\title{
Macrosegregation of Impurities in a Metallurgical Silicon Ingot After Transient Directional Solidification
}

\author{
Moyses Leite Lima ${ }^{a, b}$, Marcelo Aquino Martorano ${ }^{b *}$, João Batista Ferreira Neto ${ }^{a}$ \\ ${ }^{a}$ Laboratory of Metallurgical Processes, Institute for Technological Research, Avenida Professor \\ Almeida Prado, 532, 05508-901, São Paulo, SP, Brazil \\ ${ }^{b}$ Department of Metallurgical and Materials Engineering, University of São Paulo, Avenida Professor \\ Mello Moraes, 2463, São Paulo, SP, Brazil
}

Received: January 04, 2017; Revised: April 07, 2017; Accepted: May 25, 2017

\begin{abstract}
Metallurgical grade silicon was melted and directionally solidified in transient conditions by extracting heat from the base of a cylindrical ingot and inserting heat at the ingot top. A heat-transfer mathematical model was implemented to predict the solidification velocity and temperature gradient using cooling curves measured directly in the silicon melt. Nearly $70 \%$ of the resulting ingot displays a region of columnar grains aligned with the ingot axis. In this region, the concentration of metallic impurities is usually below the quantification limit of the analytical technique and intermetallic particles are absent, strongly indicating significant purification. The transition from the purified region of the ingot to the ingot top, where impurity concentrations increase and intermetallic particles are seen, is consistent with a change of the solid-liquid interface morphology from planar to cellular/dendritic, as similarly reported in the literature and as indicated by a preliminary analysis with the constitutional undercooling criterion.
\end{abstract}

Keywords: macrosegregation, metallurgical silicon, directional solidification, silicon refining

\section{Introduction}

The demand for silicon in photovoltaic applications has increased in the past few decades, triggering the development of several metallurgical routes to produce solar grade silicon (SoG-Si) from metallurgical grade silicon (MG-Si) ${ }^{1}$. Directional solidification is one of the most important steps in the metallurgical route ${ }^{2}$, because it causes macrosegregation of impurities and, consequently, purification of the silicon ingot. Except for boron, phosphorus, and oxygen, most of the impurities existing in MG-Si segregate to one part of the ingot, decreasing impurity concentrations in the remaining part, which becomes purified.

The macrosegregation of metallic impurities during MG-Si directional solidification in Czochralski ${ }^{3}$, Bridgman $^{4}$, and electron-beam furnaces ${ }^{5}$ has been investigated. Using the Czochralski method, Kuroda and Saitoh ${ }^{3}$ evaluated the macrosegregation for constant pulling rates from 3 to 33 $\mu \mathrm{m} / \mathrm{s}(0.2$ to $2 \mathrm{~mm} / \mathrm{min})$ and observed an abrupt increase in the impurity concentration when a cellular structure began to form. Yuge et al. ${ }^{5}$ studied the solidification of MG-Si in an electron beam furnace and found a sharp increase in the impurity concentration at the transition from a planar to a cellular/dendritic interface. In a Bridgman furnace, Martorano et al. ${ }^{4,6}$ observed that an increase in pulling velocity from 10 to $110 \mu \mathrm{m} / \mathrm{s}$ decreased macrosegregation significantly.

* e-mail: martoran@usp.br
In these studies, the solid-liquid interface velocity and temperature gradient were kept approximately constant during each experiment, requiring a relatively complex experimental technique. A simpler and more convenient directional solidification technique, referred to as transient solidification, consists of extracting heat from the ingot base, while the other ingot faces remain thermally insulated. Similar techniques are used in industrial processes such as the HEM ("heat exchanger method") ${ }^{7}$ to obtain polycrystalline silicon ingots from pure silicon feedstock. Despite the simplicity of this method, the temperature gradient and solidification velocity are not constant during solidification, evolving according to an uncontrolled and complex pattern. The effects of this specific pattern on the solid-liquid interface morphology, grain structure, and macrosegregation of impurities have not been studied in the literature.

The simplicity of the transient solidification method could be used to design a more economical directional solidification step to purify MG-Si. Therefore, the objective of the present work is to investigate the macrosegregation of metallic impurities and the type of grain structure existing in an ingot obtained from a MG-Si melt solidified directionally under transient conditions. To characterize the heat transfer conditions in the experiments, the temperature was measured in the silicon melt/ingot and was used to adjust a heat transfer mathematical model, which allowed calculations of the instantaneous solid-liquid interface velocity and temperature gradient. 


\section{Transient Directional Solidification Experiments}

A charge of $8 \mathrm{~kg}$ of MG-Si with composition obtained by ICP-OES (Table 1) was melted in a graphite-clay crucible coated with silicon nitride and located within an electric resistance $\left(\mathrm{MoSi}_{2}\right)$ furnace (Figure 1). An argon atmosphere created with a flow of 15 to $20 \mathrm{Nl} /$ min was maintained over the melt surface to prevent excessive silicon oxidation. After the charge was melted, the furnace was held for $40 \mathrm{~min}$ at $1600{ }^{\circ} \mathrm{C}$ to homogenize the molten silicon temperature at approximately $1550{ }^{\circ} \mathrm{C}$. A water-cooled copper block was brought in contact with the crucible base to extract heat from the melt and cause its transient directional solidification upwards. There were heating resistance sets near the crucible lateral wall and crucible top to control the melt cooling and solidification. The top heater set was programmed to a cooling rate of $0.3^{\circ} \mathrm{C} / \mathrm{min}$ and the lateral heater set to $0.5^{\circ} \mathrm{C} /$ min, leading to a decrease in solidification velocity and an increase in temperature gradient during solidification, which usually increase the macrosegregation of solute elements.

Two type-B (Pt-6 pct Rh / Pt-30 pct Rh) thermocouples were mounted inside alumina sheaths of $10 \mathrm{~mm}$ external diameter and inserted into the silicon melt, along the cylindrical crucible axis, to measure the cooling curves at two points during the experiment. The thermocouples were connected to a data acquisition system to register the cooling curves with a sampling rate of $2 \mathrm{~Hz}$. One thermocouple coldjunction was located at the ingot base and the other, near the melt top, at $10 \mathrm{~mm}$ below the free surface of a silicon melt of $100 \mathrm{~mm}$ height.

A longitudinal slice $40 \mathrm{~mm}$ wide and $20 \mathrm{~mm}$ thick was cut off from the center of the cylindrical ingot $(210 \mathrm{~mm}$ diameter) for macro and microstructure examination and for chemical analysis (Figure 2) to reveal macrosegregation. For the macrostructure examination, the longitudinal face of the slice was first grinded with 200, 400, 600, and 1000 mesh sandpapers and then heated up to $90{ }^{\circ} \mathrm{C}$ for etching in an aqueous solution of $\mathrm{NaOH}$. To reveal the microstructure, this same face was polished using diamond paste $(6,3$, and $1 \mu \mathrm{m})$

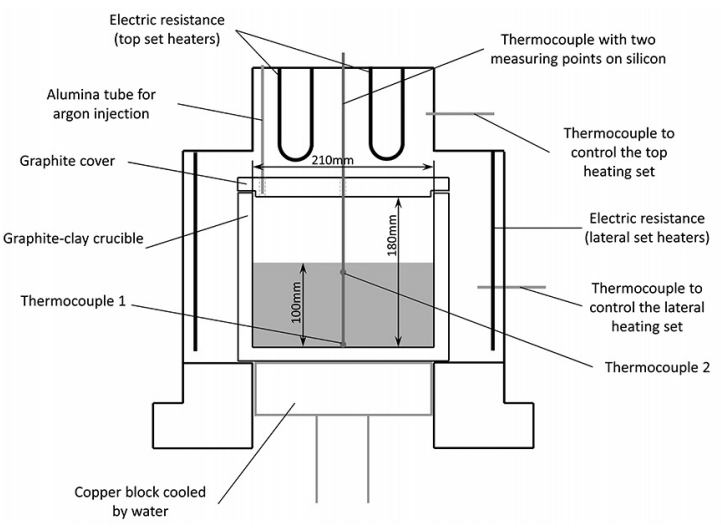

Figure 1. Schematic view of the furnace showing the crucible, water-cooled cooper block, thermocouples to control the heating sets and to measure the temperature in the molten/solid silicon.

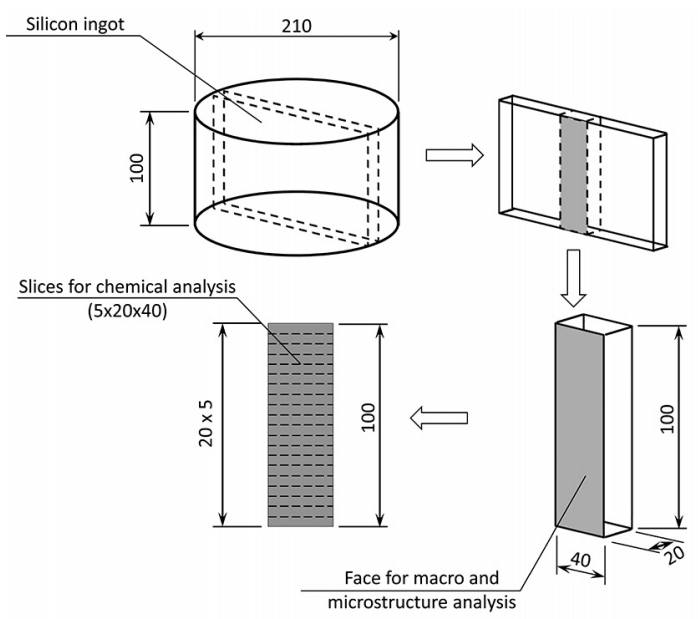

Figure 2. Scheme of sample definition for macro and microstructure examination and for chemical analysis (dimensions in $\mathrm{mm}$ ).

and finally colloidal silica. After examining the macro and microstructure, the slice was cut in 20 pieces of $5 \mathrm{~mm}$ height (Figure 2). Eight of these pieces were chosen (based on their microstructure) for chemical analysis by inductively coupled plasma mass spectrometry (ICP-MS).

Table 1. Partition coefficient between solid and liquid for solute element $i\left(k_{0 i}\right)$, diffusion coefficient of element $i$ in the liquid $\left(D_{l i}\right)$, solubility of element $i$ in solid $\mathrm{Si}(\mathrm{SL})^{4}$; and the concentration of the main metallic impurities in the MG-Si $\left(C_{0 i}\right)$ used in the experiment.

\begin{tabular}{ccccc}
\hline Element $(i)$ & $k_{0 i}$ & $D_{l i}\left(\mathrm{~m}^{2} / \mathrm{s}\right)$ & SL $(\mathrm{ppmw})$ & $C_{0 i}(\mathrm{ppmw})$ \\
\hline $\mathrm{Fe}$ & $8.0 \times 10^{-6}$ & $1.8 \times 10^{-8}$ & 0.3 & 1464 \\
$\mathrm{Ni}$ & $8.0 \times 10^{-6}$ & $1.8 \times 10^{-8}$ & 4.2 & 22 \\
$\mathrm{Ti}$ & $3.6 \times 10^{-6}$ & $1.5 \times 10^{-8}$ & 0.14 & 98 \\
$\mathrm{~V}$ & $4.0 \times 10^{-6}$ & $1.6 \times 10^{-8}$ & 0.18 & 4 \\
$\mathrm{Mn}$ & $1.0 \times 10^{-5}$ & $1.6 \times 10^{-8}$ & 0.29 & 128 \\
$\mathrm{Cr}$ & $1.1 \times 10^{-5}$ & $1.6 \times 10^{-8}$ & 0.37 & 4 \\
$\mathrm{Cu}$ & $4.0 \times 10^{-4}$ & $1.7 \times 10^{-8}$ & 1.8 & 9 \\
$\mathrm{Al}$ & $2.0 \times 10^{-3}$ & $6.8 \times 10^{-8}$ & 20 & 534 \\
\hline
\end{tabular}




\section{Mathematical Model Description}

A mathematical model is proposed and implemented to calculate the solid-liquid interface velocity and temperature gradient in the transient directional solidification experiment described in section 2. The following unidirectional heat conduction equation written for a reference system at the ingot base was solved

$$
\rho C_{P} \frac{\partial T}{\partial t}=\frac{\partial}{\partial x}\left(K \frac{\partial T}{\partial x}\right)+\rho \Delta H_{f} \frac{\partial \varepsilon_{s}}{\partial t}
$$

where $T$ is temperature; $\rho$ is density; $C_{p}$ is the heat capacity; $K$ is thermal conductivity; $\Delta H_{f}$ is latent heat per unit mass; and $\varepsilon_{S}$ is the solid fraction, which was assumed to change linearly from 0 to 1 in a narrow temperature interval between $1414^{\circ} \mathrm{C}$ (melting point of pure silicon) and $1413.9^{\circ} \mathrm{C}$. This solidification temperature interval of $0.1{ }^{\circ} \mathrm{C}$ for the MG-Si was assumed arbitrarily small to model the solidification of silicon with a very limited amount of impurities. Initially, the system was assumed a liquid of homogenous composition and uniform temperature $\left(1550^{\circ} \mathrm{C}\right)$. Eq. (1) was solved numerically using the implicit finite volume method ${ }^{8}$ with a mesh of at least 1000 volumes and a time step of $0.01 \mathrm{~s}$, using as boundary conditions the temperatures measured by two thermocouples, one located near the ingot base (thermocouple 1) and the other, near the ingot top (thermocouple 2). The thermophysical properties adopted $^{9}$ for silicon in the simulations were $K=58.2 \mathrm{~W} / \mathrm{m} \mathrm{K}$, $\rho=2560 \mathrm{~kg} / \mathrm{m}^{3}, C_{P}=968 \mathrm{~J} / \mathrm{kg} \mathrm{K}$, and $\Delta H_{f}=1.787 \times 10^{6} \mathrm{~J} / \mathrm{kg}$.

\section{Experimental Results}

The two cooling curves measured in the experiments and used as boundary conditions of the mathematical model are given in Figure 3. The reference time $(t=0)$ is the moment when the water-cooled copper block contacted the crucible bottom and began to extract heat. Between 7.5 and $8.5 \mathrm{~h}$ of cooling, an abrupt decrease in temperature is observed in the thermocouple 2, which probably indicates the end of the ingot solidification.

The grain structure of the resulting ingot (Figure 4a) consists essentially of columnar grains aligned with the ingot axis (solidification direction), as generally expected in directionally solidified ingots. At about $70 \mathrm{~mm}$ from the ingot bottom, there is a structure transition, above which grains display serrated boundaries and are not clearly columnar or aligned. Yuge et al. ${ }^{5}$, Kuroda and Saitoh ${ }^{3}$ observed similar grain structure transitions, which were considered as a transition of solid-liquid interface morphology from planar to cellular/dendritic. Therefore, the serrated boundaries could be a result of a cellular/dendritic interface morphology.

The microstructures of the ingot samples were examined with an optical (Figure 4b, c, d, and e) and scanning electron microscopes (Figure 4f). Intermetallic particles were seen only in the samples from above the grain structure

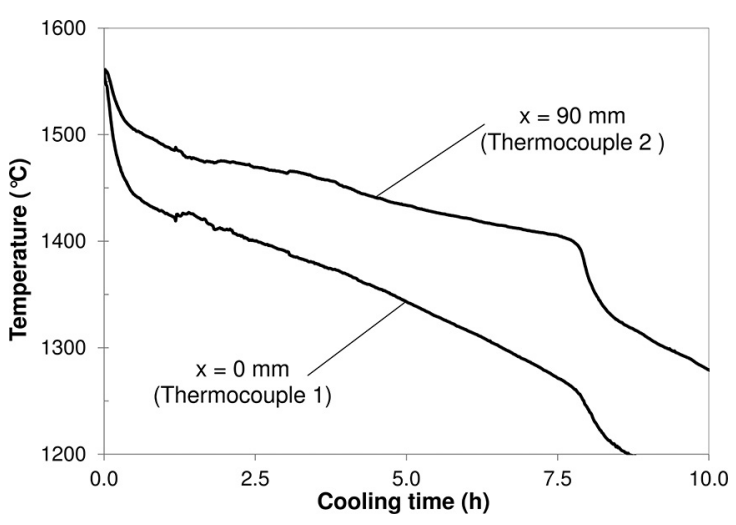

Figure 3. Cooling curves measured directly in the silicon melt during the transient directional solidification experiment.

transition. Examples of microstructures from above and below this transition are illustrated in Figure 4. Details of one particle found in a region at $75 \mathrm{~mm}$ from the ingot base (Figure 4c) is shown in Figure 4f. Four EDS (energy dispersive spectroscopy) microanalyses were carried out in the spots shown within this particle, indicating the presence of mainly Si, Fe, Mn, and Ti.

In Figure 5, the macrosegregation profiles of $\mathrm{Fe}, \mathrm{Al}$, $\mathrm{Mn}$, and Ti measured by ICP-MS as a function of the relative position in the axial direction, $\mathrm{x} / \mathrm{L}$ ( $\mathrm{x}$ is the distance from ingot base and $\mathrm{L}$ is the ingot length), are given. The solubility limit (SL) of each element in solid Si (Table 1) and the quantification limits (QL) of the chemical analyses are indicated for each impurity. The concentration profiles show a macrosegregation of impurity elements towards the ingot top $(\mathrm{x} / \mathrm{L}=1)$, which was the last region to solidify. This type of macrosegregation profile is usually observed after solidification with a planar solid-liquid interface, because when solidification occurs with a dendritic/cellular interface, solute transport from the mushy zone into the bulk liquid is hindered, decreasing or even eliminating macrosegregation. Note that the relative concentration of the impurities are significantly below the initial composition of the MG-Si melt $\left(C_{i} / C_{0 i}=1\right)$ along $70 \%$ of the ingot height, indicating an important purification effect achieved with the transient solidification conditions.

The position where the first intermetallic compounds were found in the microstructure (Figure 4c) coincides with the abrupt increase in impurity concentrations above the solubility limit seen in Figure 5. Moreover, it also coincides with the transition in grain structure indicated by the dashed line in Figure 4a. Therefore, the increase in impurity concentration, the formation of intermetallic particles, and the change in grain morphology are all related. These observations are consistent with the results of Yuge et al. ${ }^{5}$, who associated the macrostructure transition and the appearance of intermetallic particles with the interface morphology transition from planar to cellular/dendritic. 


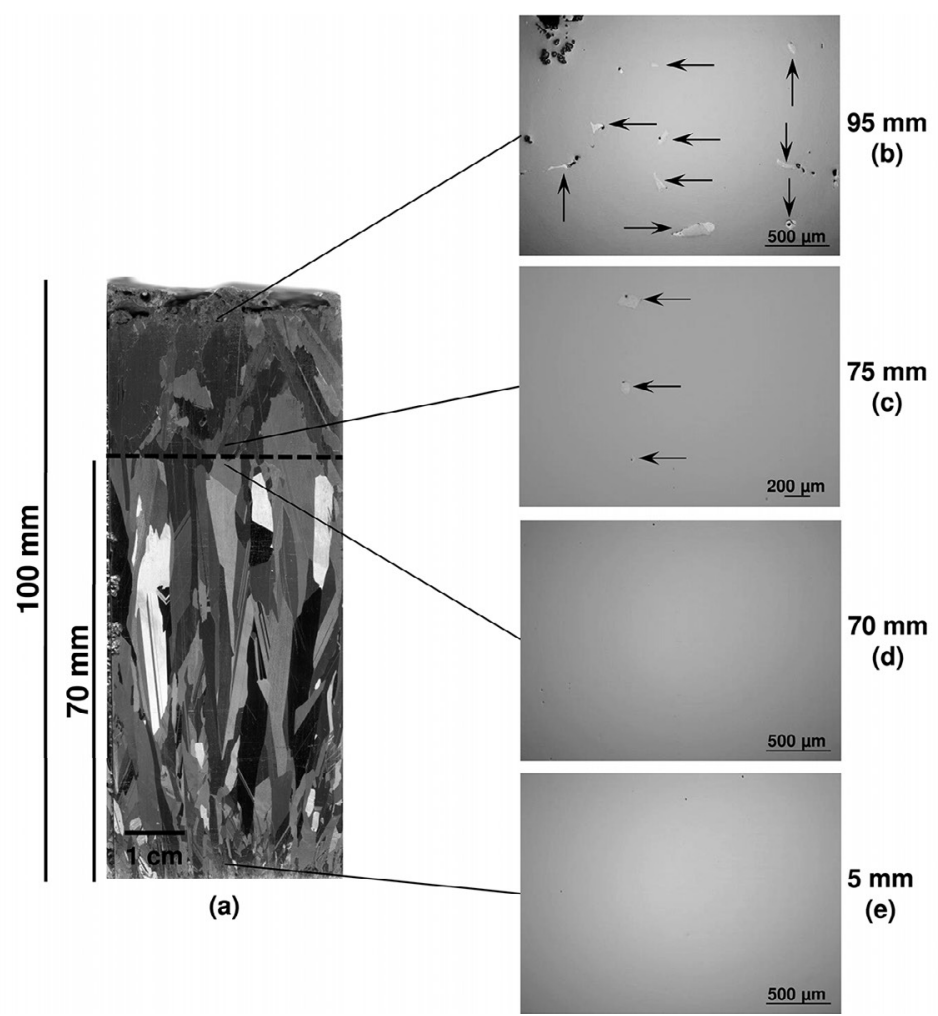

Figure 4. Metallurgical structure of the resulting ingot (central portion): (a) macrograph (etchant: $\mathrm{NaOH}$ solution at $90^{\circ} \mathrm{C}$ ) and $(\mathrm{b}, \mathrm{c}, \mathrm{d}, \mathrm{e})$ micrographs of the longitudinal section, with arrows pointing to intermetallic particles (no etching, optical microscopy), from different positions relative to the ingot base (indicated on the right). Details of an intermetallic found in (c) are given in (f), showing four spots where EDS microanalyses were carried out (scanning electron microscopy with backscattered electron).
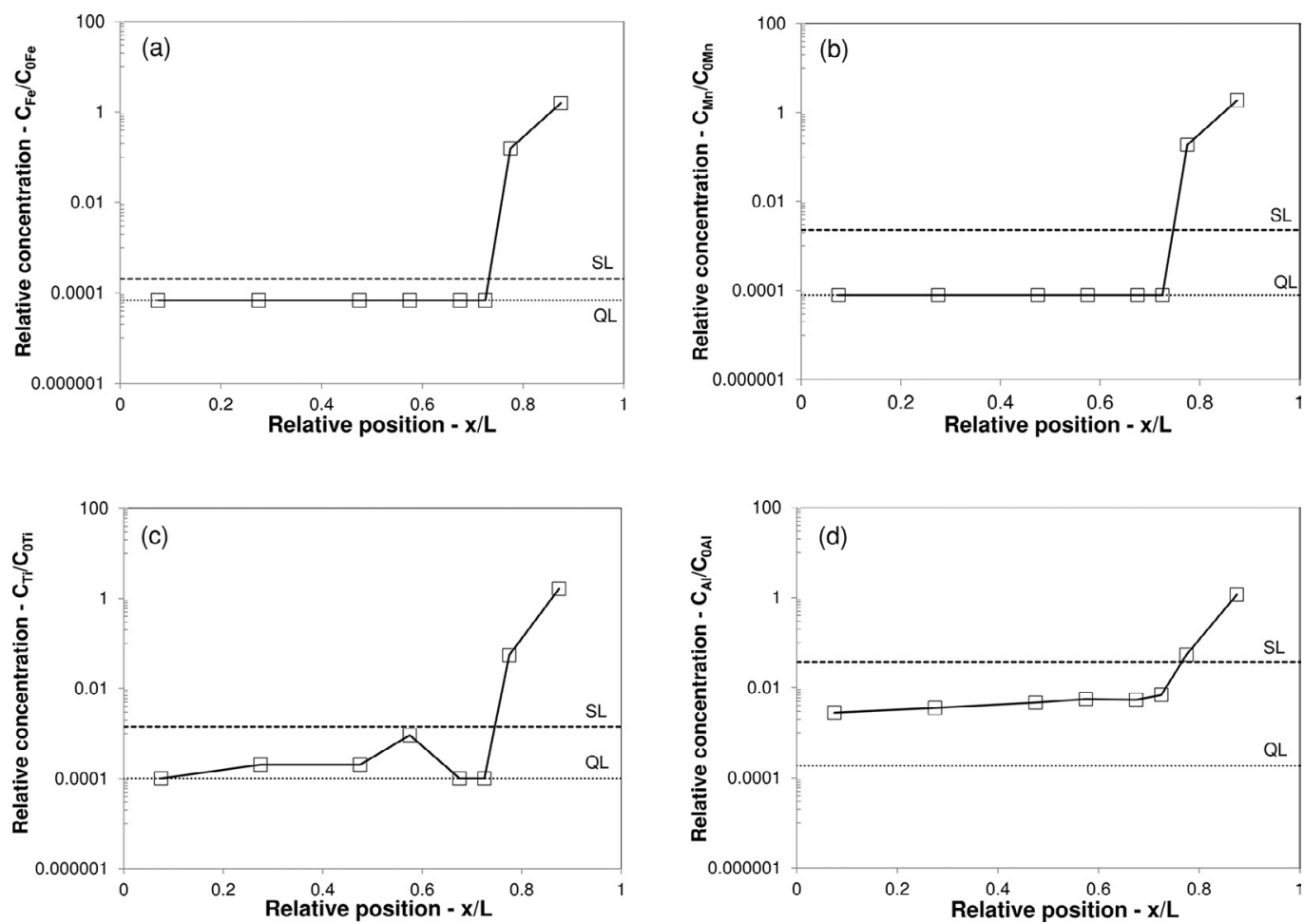

Figure 5. Profiles of relative concentration measured by ICP-MS along the axial direction at the ingot center for (a) Fe, (b) $\mathrm{Mn},(\mathrm{c}) \mathrm{Ti}$, and (d) $\mathrm{Al}$ as a function of the relative position in relation to the ingot base. The solubility limit (SL) of each element in solid Si and the quantification limit of the ICP analyses (QL) are also shown. 
Therefore, below the dashed line $(\mathrm{x} \sim 70 \mathrm{~mm})$ indicated in Figure $4 \mathrm{a}$, the ingot probably solidified with a planar solidliquid interface, whereas above the line it solidified with a dendritic/cellular interface.

\section{Discussion}

The solid-liquid interface velocity $(V)$ and the temperature gradient $(G)$ at the liquid adjacent to the interface were calculated with the present mathematical model as a function of the interface position (Figure 6), after calculating the temperature field. The transient character of the directional solidification experiment is evident from the variations of $V$ and $G$ during solidification. Average values of $V \sim 5 \mu \mathrm{m} / \mathrm{s}$ and $G \sim 800 \mathrm{~K} / \mathrm{m}$ are observed until approximately $70 \%$ of the ingot has solidified. Thereafter, $V$ decreases and $G$ increases. The observed values and variations of $V$ and $G$ are a result of the particular heat input during solidification from the heating elements located near the crucible wall and top. Without this heat input, a larger $V$ and a lower $G$ would be expected, which would favor the formation of a mushy zone and a consequent decrease in macrosegregation. Therefore, an understanding of the conditions to stabilize the planar solid-liquid interface is needed to increase macrosegregation and purification.

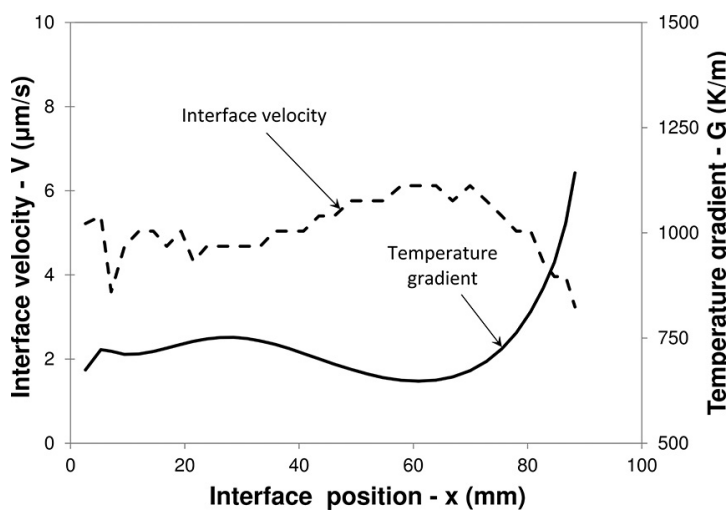

Figure 6. Mathematical model results for the interface velocity $(V)$ and the temperature gradient $(G)$ at the liquid adjacent to the solid-liquid interface as a function of the interface position along the axial direction.

The stability of a planar solid-liquid interface was evaluated in the present work conditions by application of the constitutional supercooling criterion (CSC), as described by Martorano et al. ${ }^{4}$, using the following equation

$$
\frac{G}{V}>\mathrm{CSC}=-\sum_{i=1}^{N}\left[\frac{m_{l i} C_{s i}\left(1-k_{0 i}\right)}{D_{l i} k_{0 i}}\right]
$$

where $i$ is a solute element; $N$ is the total number of solute elements considered in the analysis $(N=8) ; m_{l i}$ is the liquidus line slope obtained from the phase diagram for the binary system of $\mathrm{Si}$ and element $i$; $C_{s i}$ is solute concentration in the solid adjacent to the solid-liquid interface during solidification; $k_{0 i}$ is the solute partition coefficient for element $i$, also obtained from the binary phase diagram; and $D_{l i}$ is the diffusion coefficient of element $i$ in the liquid. Some of these parameters are given in Table 1 . The values of $G$ and $V$ were calculated with the present mathematical model (Figure 6).

Considering that the diffusion of elements in solid $\mathrm{Si}$ is negligible, the values of $C_{s i}$ at the planar solid-liquid interface are maintained in the solid after the end of solidification and, therefore, can be obtained from the measured macrosegregation profiles of Fe, Mn, Ti, Al, Ni, V, Cr and Cu. Some of these profiles are given in Figure 5, showing that many of the concentration measurements are below the quantification limit (QL) of the analytical technique. For these measurements, two different values of $C_{s i}$ were adopted to calculate the CSC parameter in the right-hand side of Eq. (2): one is QL and the other is the concentration calculated from Scheil model $^{10}$, given below

$$
C_{s i}=k_{0 i} C_{0 i}(1-x / L)^{\left(k_{0 i}-1\right)}
$$

Note that the actual value of $C_{s i}$ should be in the range between QL and that given by Scheil model, in which complete mixing of liquid composition is assumed. To illustrate these concentrations, the measured macrosegregation profile, the $\mathrm{QL}$, and the concentration given by Scheil equation for $\mathrm{Fe}$ are shown in Figure 7.

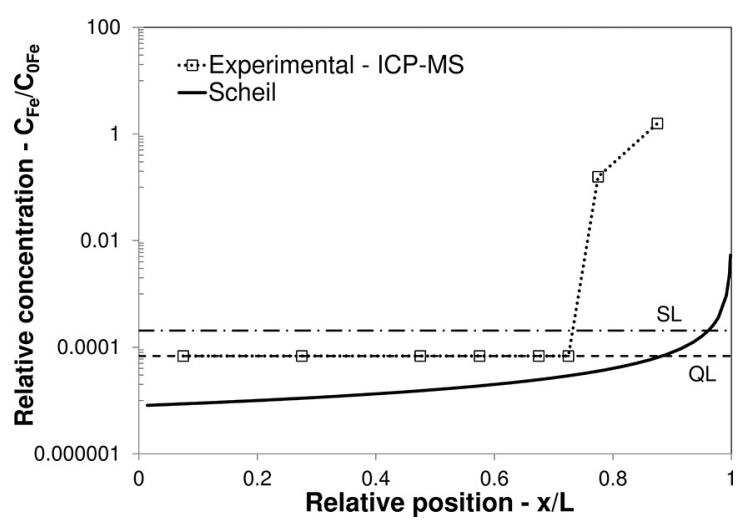

Figure 7. Profiles of relative concentration of Fe measured by ICP-MS and calculated with Scheil equation. The solubility limit (SL) of the element in the solid phase and the quantification limit of the ICP-MS analyses (QL) are also shown.

Figure 8 shows calculations of $\mathrm{G} / \mathrm{V}$ and of the $\mathrm{CSC}$ parameter (Eq. 2) using two different values for : (i) the measured concentrations (macrosegregation profile) when they are above the QL or the QL otherwise (CSC_ICP-MS); (ii) the concentrations calculated with the Scheil equation, Eq.(3) (CSC_Scheil). According to Eq.(2), the solidliquid interface is planar when $\mathrm{G} / \mathrm{V}$ is larger than the $\mathrm{CSC}$ parameter, implying that there would be no planar interface for the CSC_ICP-MS calculation and that the interface would be planar from the ingot base up to $\mathrm{x} \sim 82 \mathrm{~mm}$ for the CSC_Scheil calculation. As discussed in section 4, the 


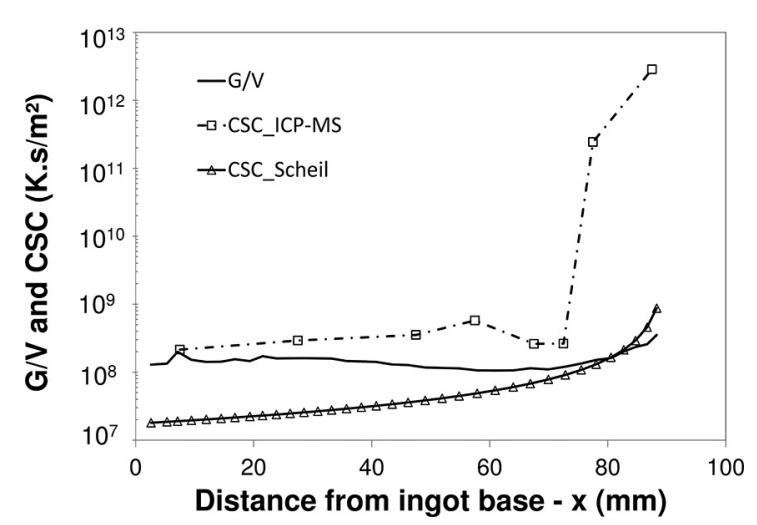

Figure 8. Calculations of $G / V$ and of the CSC parameter for measured concentrations (ICP-MS) and Scheil model concentrations (CSC-Scheil) as a function of the distance from the ingot base (x).

micro and macrostructure of the ingot indicate solidification with a planar solid-liquid interface up to $\mathrm{x} \sim 70 \mathrm{~mm}$, which is in between the values given by CSC_ICP-MS $(\mathrm{x}=0)$ and CSC_ICP-MS $(\mathrm{x} \sim 82 \mathrm{~mm})$. To be consistent with this interval, the actual unknown CSC line (based on the actual unknown $C_{s i}$ value) should lie between the CSC_ICP-MS and CSC_Scheil lines to give a planar interface up to $\mathrm{x} \sim$ $70 \mathrm{~mm}$. This seems to occur, because the actual $C_{s i}$ value is certainly below the QL and probably above the value given by Scheil model, as explained in the next paragraph.

In the Scheil model, complete mixing of the liquid composition is assumed, representing the fastest possible transport of solute from the solid-liquid interface into the bulk liquid. This fast solute transport decreases the concentration in the solid $\left(C_{s i}\right)$ to its minimum possible value and increases macrosegregation to its maximum value. The hypothesis of complete liquid mixing in Scheil model is consistent with a vigorous forced liquid convection. In the present experiment, however, there was less vigorous thermal convection, probably due only to radial temperature gradients. Therefore, macrosegregation would be less intense and the actual $C_{s i}$ would be larger than the $C_{s i}$ given by Scheil equation (Eq. 3).

Approximately $70 \%$ of the ingot has been significantly purified in relation to the initial MG-Si composition $\left(C_{0 i}\right)$ for the present transient solidification technique, which is simpler than other directional solidification methods, such as the Bridgman directional solidification. The solidification velocity prevailing during solidification of the purified region of the ingot was approximately $5 \mu \mathrm{m} / \mathrm{s}$, which is similar to the velocity reported by Martorano et al. ${ }^{4}$ for significant purification in the Bridgman furnace. The temperature gradient calculated in the present work $(\mathrm{G} \sim 800 \mathrm{~K} / \mathrm{m})$, however, is lower than those observed by Martorano et al. ${ }^{4}$ and by Kuroda et al. $^{3}(\mathrm{G} \sim 2000 \mathrm{~K} / \mathrm{m})$, but similar to that reported by by Yuge et al. ${ }^{5}(750 \mathrm{~K} / \mathrm{m}<G<1000 \mathrm{~K} / \mathrm{m})$.

\section{Conclusions}

Metallurgical grade silicon was melted and directionally solidified by extracting heat from the base of a cylindrical ingot and inserting heat at the ingot top to control solidification conditions. This technique is called transient directional solidification, in which the solidification velocity and temperature gradient are neither constant nor directly controlled. This technique is usually simpler than traditional techniques, such as the Bridgman furnace solidification. Nearly $70 \%$ of the resulting cylindrical ingot (100 mm height; $210 \mathrm{~mm}$ diameter) consists of columnar grains approximately aligned with the heat extraction flux, whereas the remaining ingot consists of grains with serrated boundaries and no apparent common alignment direction. The columnar grain structure coincides with a region of solute concentrations usually below the quantification limit of the analytical technique and without intermetallic particles, indicating a significantly purified region in comparison with the metallurgical silicon composition. The observed grain structure transition was also reported by different authors ${ }^{3,5}$ and is consistent with a transition of the solid-liquid interface morphology from planar (columnar aligned grains) to cellular/dendritic during ingot solidification. The position of the transition region is also consistent with a preliminary analysis carried out using the constitutional supercooling criterion. Finally, the present work results indicate that $70 \%$ of an ingot formed from a metallurgical grade silicon melt can be efficiently purified by a transient directional solidification technique.

\section{Acknowledgements}

Authors thank the support from Companhia Ferro-Ligas Minas Gerais (Minas Ligas), National Bank for Social and Economic Development (BNDES), and Conselho Nacional de Desenvolvimento Científico e Tecnológico (CNPq) (311206/2014-0).

\section{References}

1. Pizzini S. Towards solar grade silicon: Challenges and benefits for low cost photovoltaics. Solar Energy Materials \& Solar Cells. 2010;94(9):1528-1533.

2. Safarian J, Tranell G, Tangstad M. Processes for upgrading metallurgical grade silicon to solar grade silicon. Energy Procedia. 2012;20:88-97.

3. Kuroda E, Saitoh T. Growth and characterization of polycrystalline silicon ingots from metallurgical grade source material. Journal of Crystal Growth. 1979;47(2):251-260.

4. Martorano MA, Ferreira Neto JB, Oliveira TS, Tsubaki TO. Macrosegregation of Impurities in Directionally Solidified Silicon. Metallurgical and Materials Transactions A. 2011;42(7):1870-1886. 
5. Yuge N, Hanazawa K, Kato Y. Removal of Metal Impurities in Molten Solicon by Directional Solidification with Electron Beam Heating. Materials Transactions. 2004;45(3):850-857.

6. Martorano MA, Ferreira Neto JB, Oliveira TS, Tsubaki TO. Refining of metallurgical silicon by directional solidification. Materials Science and Engineering B. 2011;176(3):217-226.

7. Khattak CP, Basaran M, Schmid F, D'aiello RV, Robinson PH, Firester AH. Metallurgical silicon substrateproduced by HEM for epixitaxial thin films solar cells. In: Proceedings of $15^{\text {th }}$
IEEE Photovoltaic Specialists Conference; 1981 May 12-15; Orlando, FL, USA. p. 1432.

8. Patankar SV. Numerical heat transfer and fluid flow. New York: Hemisphere Publishing; 1980

9. Mills KC, Courtney L. Thermophysical properties of silicon. ISIJ International. 2000;40(Suppl P):S130-S138.

10. Flemings MC. Solidification Processing. New York: McGrawHill; 1974. 